\title{
LA LUCHA INSTITUCIONAL CONTRA LA PROPAGANDA DE LAS GRANDES EMPRESAS ESTADOUNIDENSES DURANTE LA GRAN DEPRESIÓN ${ }^{1}$
}

\author{
THE INSTITUTIONAL STRUGGLE AGAINST THE PROPAGANDA \\ CAMPAIGNS OF LARGE AMERICAN COMPANIES \\ DURING THE GREAT DEPRESSION
}

\author{
Dario Migliucci* \\ Universidad Complutense de Madrid, España - Sorbonne Universitè, París, Francia
}

\begin{abstract}
RESUMEN: El objetivo del presente trabajo es el de analizar la labor de los comités legislativos estadounidenses que fueron establecidos, durante la década de los treinta del siglo XX, con el fin de indagar la propaganda de las grandes corporaciones privadas. En particular, se analizarán las investigaciones sobre las empresas de gas y electricidad, las industrias armamentísticas y las major cinematográficas. La hipótesis es que, si bien muchos dirigentes fueron movidos por la genuina voluntad de combatir abusos y malas prácticas, a largo plazo las investigaciones acabaron siendo dominadas por una acentuada radicalización ideológica y por una destacada deriva emocional, lo que a la postre frustró la posibilidad de que, a partir de la labor de los comités, pudiesen surgir medidas de regulación del sector.
\end{abstract}

PALABRAS CLAVE: Propaganda, Estados Unidos de América, Empresas privadas, Comités legislativos de investigación.

ABSTRACT: The aim of the article is to analyze the nature of the committees that were established in the 1930s in the United States of America in order to investigate the propaganda campaigns of private corporations. In particular, the research will focus on the investigations into propaganda activities of gas and electricity companies, arms industries and film studios. The hypothesis is that, although many politicians were moved by a genuine willingness to ensure justice, in the long term the investigations would end up being dominated by ideological radicalization and emotional drift, which ultimately frustrated the possibility that, from the work of the committees, regulatory measures of the financial sector could arise.

KEYWORDS: Propaganda, United States of America, Private Companies, Legislative Committee Investigations.

1 Una versión preliminar del presente artículo ha formado parte de la tesis doctoral con Mención Internacional «La propaganda como desafío para la democracia. La clase política estadounidense y la manipulación de la opinión pública (1919-1941)», defendida en la Universidad Complutense de Madrid el 31 de octubre de 2019. La investigación ha sido financiada por el Ministerio de Educación a través de una ayuda FPU (FPU14/01884).

* Correspondencia a / Corresponding author: Dario Migliucci, Universidad Complutense de Madrid, Facultad de Geografía e Historia, Edif. B, Calle del Prof. Aranguren, s/n, 28040 Madrid, España - dariomigliucci@hotmail.com - https://orcid.org/0000-0002-8753-9716

Cómo citar / How to cite: Migliucci, Dario (2022). "La lucha institucional contra la propaganda de las grandes empresas estadounidenses durante la Gran Depresión», Historia Contemporánea, 68, 171-202. (https://doi.org/10.1387/hc.21442).

Recibido: 2 febrero, 2020; aceptado: 26 mayo, 2020.

ISSN 1130-2402 - eISSN 2340-0277 / (c) 2022 Historia Contemporánea (UPV/EHU) 
Como es bien sabido, los años treinta del siglo Xx fueron marcados por una crisis económica global de proporciones catastróficas. En los Estados Unidos de América los llamados Roaring Twenties - una década caracterizada por una orgía consumista sin precedentes - terminaron abruptamente en octubre de 1929, cuando una gigantesca burbuja especulativa culminó con el derrumbe de la Bolsa de Nueva York. Fue aquello el comienzo de un largo periodo de depresión económica, durante el cual un enorme número de trabajadores perdió su empleo, acabando millones de familias en condiciones de absoluta miseria. La confianza de la ciudadanía hacia las grandes empresas, lógicamente, se desplomó. El clima de desesperación degeneró frecuentemente en sentimientos de odio y rencor, al imputarse al sector empresarial la responsabilidad de todas las desgracias que las clases populares estaban soportando.

En dicho contexto de emergencia nacional, no puede extrañar que la clase política se sintiese en la obligación de impulsar respuestas institucionales que pudiesen ser percibidas por la opinión pública como contundentes y resolutorias. En estos últimos años - seguramente a raíz de las inquietudes generadas por la Gran Recesión que se originó en 2008varios investigadores han posado su mirada en el célebre comité del Senado que, entre 1932 y 1934, indagó los abusos de Wall Street, una investigación que derivó finalmente en la promulgación de medidas tan importantes como la Glass-Steagall Banking Act (1933) y la Securities Exchange Act (1934), que regularían el sistema bancario y bursátil durante las décadas siguientes ${ }^{2}$. En cuanto al presente trabajo, su objetivo es el de analizar la labor de los numerosos comités legislativos que fueron establecidos, a lo largo de la década de los treinta, con el fin de indagar las campañas de propaganda de las grandes corporaciones privadas. Para ello, se examinarán fuentes primarias que han sido recolectadas en la National Archives and Records Administration de College Park (Maryland) y Washington D.C. y en la Biblioteca del Congreso de la capital estadounidense.

La aprensión relativa a las actividades de manipulación de la opinión pública se había originado ciertamente durante la Primera Guerra Mundial, cuando el Committee on Public Information - el primer aparato propagandístico institucional de la historia de los Estados Unidos - había conseguido construir un fuerte consenso popular entorno a la causa de

2 Por ejemplo, Perino, 2010; Moss, Bolton y Kintgen, 2010; y Derbyshire, 2012. 
la intervención militar ${ }^{3}$. Tras el fin del conflicto, muchos autores alertaron sobre la posibilidad de que ciertas estrategias de manipulación masiva pudiesen ser empleadas por las empresas privadas con el fin de promover sus intereses económicos ${ }^{4}$. No se trataba por supuesto de renegar de las actividades publicitarias - una práctica comercial plenamente aceptada en la sociedad de la época - sino de denunciar operaciones de propaganda engañosas u ocultas, cuya finalidad era la de crear un clima de opinión favorable a los objetivos estratégicos de las grandes corporaciones.

Se sospechaba por ejemplo que se realizasen fichajes encubiertos de periodistas y escritores, lográndose de esta manera que diarios y libros escolares promoviesen - aparentemente de forma espontánea y desinteresada - las ventajas de la gestión privada de determinados servicios públicos (energía, ferrocarril, etc.) frente a su administración por parte del Estado. También se planteaba la posibilidad de que el envío masivo a los congresistas de cartas y telegramas por parte de ciudadanos de a pie - documentos en los que se manifestaba profunda inquietud por la promulgación de medidas intervencionistas - fuese en realidad obra de redes de individuos a sueldo de alguna empresa. En suma, la diferencia entre lo que se consideraba como una legítima campaña de publicidad y lo que se tachaba de propaganda ilícita descansaba básicamente en la cuestión de su trasparencia.

Aunque la bonanza económica de la década de los veinte consiguió que los recelos relativos a los engaños de los colosos de la economía se disipasen temporalmente, con la llegada de la Gran Depresión dichas inquietudes volvieron a encontrar un sitio privilegiado en el debate público. A lo largo de toda la década de los treinta, y hasta la entrada del país en la Segunda Guerra Mundial, se sucedieron en los Estados Unidos un gran número de investigaciones legislativas sobre la propaganda de los privados. Establecidos en los más altos niveles de las instituciones de la República, los distintos comités pusieron el foco en las actividades de organizaciones tan heterogéneas como las empresas que gestionaban los servicios de gas y electricidad, las industrias de las armas o las major cinematográficas.

La hipótesis que será defendida a lo largo de este trabajo es que, si bien muchos dirigentes fueron movidos por la genuina voluntad de depurar responsabilidades - señalando los fallos estructurales y las malas prácticas que habían contribuido a provocar el desastre económico de

${ }^{3}$ Sobre el aparato propagandístico de la Gran Guerra, véase Creel, 1920; Mock y Larson, 1939; y Vaughn, 1980.

${ }_{4}^{4}$ Migliucci, 2016; 2018A; y 2018B. 
aquellos años - a largo plazo las actividades de investigación del Congreso acabarían siendo dominadas por una cada vez más acentuada radicalización ideológica y por una destacada deriva emocional. Al mezclarse con las crecientes pasiones populares de la época, las investigaciones fueron asumiendo paulatinamente las características propias de un fenómeno de irracionalidad colectiva. De esta manera, la labor de los congresistas perdió credibilidad, frustrándose a la postre la posibilidad de que, a partir de las investigaciones de los comités, pudiesen surgir nuevas medidas de regulación de los sectores empresarial y financiero.

\section{La larga lucha del Estado contra los abusos de las empresas}

Debido a las graves turbulencias sociales ocasionadas por la Gran Depresión, en la década de los treinta retomaron vigor en los Estados Unidos ansiedades que habían estado en el centro del debate político durante los últimos años del siglo XIX. Por una parte, se reavivó el temor por la concentración de poder económico en las manos de las grandes corporaciones. Por otra, volvió a generar profunda inquietud la manera en la que los colosos de la economía lograban moldear las actitudes de los ciudadanos. Las medidas con las que se planteaba reprimir los comportamientos improcedentes solían reabrir antiguas querellas sobre cuestiones ideológicas que nunca habían gozado de un consenso unánime entre la opinión pública. ¿Hasta qué punto se podían limitar - en el país de la libertad de expresión y del laissez faire - las campañas propagandísticas de las empresas? ¿En qué medida las leyes promulgadas en el Distrito de Columbia representaban una amenaza para las prerrogativas de los estados y las libertades de los ciudadanos? Y, sobre todo, ¿quién establecía el límite entre lo que debía considerarse como una legítima promoción de ideas y de productos y lo que tenía que tacharse de propaganda?

A caballo entre los siglos XIX y XX largos debates y enredados compromisos llevaron a la promulgación de una legislación finalizada a limitar los abusos del sector, procurándose que fuesen preservadas al mismo tiempo las libertades fundamentales de las empresas ${ }^{5}$. Un hito en este sentido fue la Sherman Act (1890), con la que se limitaron los monopolios ${ }^{6}$.

\footnotetext{
5 Sugár, 2011.

${ }^{6}$ En 1914 la legislación sería reforzada con la Federal Trade Commission Act y la Clayton Act.
} 
Por su parte, la Newspaper Publicity Act (1912) obligó a los periódicos a revelar la identidad de sus propietarios, dejándose así en evidencia las razones por las que muchos periodistas habían estado defendiendo determinadas causas ${ }^{7}$. La Federal Trade Commission Act (1914), por su parte, ilegalizó los anuncios fraudulentos. Otra grave preocupación de la época fueron las actividades de presión (lobby) que los grandes intereses económicos ejercían sobre los políticos. La Tillman Act (1907) y la Federal Corrupt Practices Act (1910) restringieron la posibilidad de que las corporaciones pudiesen ofrecer contribuciones económicas en ámbito electoral ${ }^{8}$.

Graves ansiedades por la propaganda ilícita de las empresas se desarrollaron durante el primer conflicto mundial, y estuvieron relacionadas principalmente con las actividades mediáticas que podían favorecer los intereses del enemigo alemán. En septiembre de 1918 el Senado puso en marcha una investigación - dirigida por el demócrata Lee Slater Overman - sobre las campañas de manipulación de las industrias del licor, que se hallaban casi todas en manos de empresarios de origen germano'. En la resolución que había llevado a su establecimiento, se mencionaba la posibilidad de que las industrias de la cerveza hubiesen estado controlando la legislación a través de la financiación oculta de numerosos órganos de prensa y de las campañas electorales de candidatos complacientes ${ }^{10}$. La idea de que las empresas de los ciudadanos de sangre alemana difundiesen propaganda hostil era entonces bastante arraigada, defendiéndose abiertamente que la industria del licor mantenía bajo su poder a los principales partidos políticos y al mismo Gobierno federa ${ }^{11}$.

El impacto de la propaganda en aquellos años fue desmesuradamente engrandecido, y tras el fin de la guerra numerosos autores manifestaron tener graves inquietudes en cuanto a las actividades de las grandes corporaciones. Uno de los más activos en este sentido fue el periodista Frank Cobb: «Private propaganda (...) has taken on new phases since the war

${ }^{7}$ La misma ley estableció que los periódicos tenían que separar claramente el contenido informativo del publicitario.

${ }^{8}$ Con todo, será sólo en 1946 cuando se aprobará un estatuto federal consagrado a regular de forma más exhaustiva las actividades de lobby.

9 Johnson, 1999.

10 «Authorizing and Directing the Committee on the Judiciary of the Senate to Call for certain Evidence and Documents relating to Charges Made against the United States Brewers Association and Allied Interests and to Report to the Senate», Senate Resolution (en adelante, S. Res.) 307, 65. ${ }^{\circ}$ congreso, 19 de septiembre 1918.

11 Thieme, ¿1918?. 
(...) [It] had extended himself to Wall Street, to big business and to most of the institutions that have to deal with public opinion». Más allá de las campañas fraudulentas, Cobb denunciaba el fenómeno de la multiplicación de los agentes de prensa: «The great corporations have them, the banks have them, the railroads have them, all the organizations of business and of social and political activity have them and they are the media through which news comes» ${ }^{12}$.

Los políticos también comenzaron a interesarse por esta problemática. El senador Charles Thomas, por ejemplo, aseguró en 1920 que el Congreso se encontraban asediado por propagandistas a sueldo de los grandes colosos de la economía. El demócrata se quejaba de que la propaganda se había convertido en un «asunto grave» en el Distrito de Columbia, ejerciendo un gran número de propagandistas graves presiones sobre los congresistas: «The only lobby not present here is a lobby of taxpayers» ${ }^{13}$. Mientras tanto, en la Cámara de los Representantes el demócrata Tom D. McKeown avanzaba una propuesta de ley cuya finalidad era la instituir un registro que permitiese tener bajo control las operaciones finalizadas a «influenciar, a través de la propaganda, la legislación nacional o la opinión pública» ${ }^{14}$.

Durante los años veinte, la preocupación por las tareas de manipulación de la opinión pública se atenuó considerablemente. Las grandes empresas repentinamente parecían inmunes a las críticas, ya que muchos ciudadanos atribuían a los productores de los bienes de consumo el mérito por la mejora de sus condiciones de vida. En 1924, por ejemplo, muy poca atención recibió la investigación de la Comisión Interestatal de Comercio del Senado sobre las empresas que, entre diciembre de 1917 y marzo de 1920, habían estado gestionando los servicios de ferrocarril. Según los senadores, dichas empresas habían difundido campañas en los medios de comunicación para convencer a la ciudadanía de las grandes ventajas que la administración privada del trasporte ferroviario les estaba otorgando a viajeros y contribuyentes ${ }^{15}$.

12 Cobb, 1919.

13 Morrow, James B., «Washington Is Filled with Propaganda», Boston Daily Globe, 29 de febrero de 1920.

14 «Requiring the Filing of certain Information by Associations, Societies, Leagues, Gatherings, or Individuals who Seek to Influence Legislation or Public Opinion by Means of Propaganda or Written Communication from their Officers, Members, or Friends», House Resolutions (en Adelante, H.R. ) 12378, 66..$^{\circ}$ congreso, 6 de febrero 1920.

15 Congressional Record-Senate, 15 de enero 1924, p. 984. 
En los años veinte, de todos modos, la principal preocupación de los políticos estuvo más bien relacionada con el creciente poder de las corporaciones radiofónicas. A caballo entre los siglos XIX y XX, los medios de comunicación habían alcanzado cotas de poder nunca vistas, generándose un conflicto abierto entre el mundo político y la prensa. Se trataba básicamente de establecer quien se erigiría como la fuerza hegemónica en cuanto a la capacidad de conformar la opinión de los electores. Dicha pugna alcanzó su apogeo durante la Progresive Era, teniendo como principales protagonistas al poderoso grupo editorial Hearts y a la administración republicana de Theodore Roosevelt ${ }^{16}$. En el periodo de entreguerras esta rivalidad se reavivó debido al estrepitoso éxito de la radio. El pueblo estadounidense había mostrado gran entusiasmo con la aparición de las programaciones comerciales, lo que les otorgaba a las emisoras un inmenso poder. El secretario al Comercio, Herbert Hoover, resolvió imponer una reglamentación del sector, un sistema de licencias otorgadas por el Gobierno que se materializó con la promulgación de la Radio Act de 1927.

Será sin embargo el crac bursátil de 1929 el gran detonante de la deriva emocional e ideológica que acabó caracterizando la respuesta de la clase política estadounidense al desafío de la propaganda privada. Cuando Franklin Delano Roosevelt ganó las elecciones (noviembre de 1932), la confianza en el libre mercado había caído a mínimos históricos. La quiebra de miles de empresas -incluidos grandes colosos como el imperio de la electricidad Insull - convenció a los ciudadanos de que la economía había sido gestionada con superficialidad e incompetencia ${ }^{17}$. El programa intervencionista del nuevo presidente fue visto por muchos como el antídoto al sistema del laissez faire. A la indignación por los abusos de las empresas le siguió el desenfrenado entusiasmo con el que muchos políticos abrazaron el proyecto político de la nueva administración demócrata.

\section{La propaganda de los colosos energéticos y el viraje hacia el intervencionismo}

Tras la caída de la bolsa de Nueva York el tema de los abusos de las empresas se encontró repentinamente en el centro de la atención general.

16 Neuzil, 1996.

17 Sobre la caída del imperio Insull, véase Cudahy y Henderson, 2005, p. 35. 
Medios de comunicación y congresistas comenzaron a otorgarles gran importancia a las revelaciones de la Federal Trade Commission, que por aquel entonces estaba examinando las modalidades con las que las corporaciones de gas y electricidad manipulaban las percepciones de la opinión pública con el fin de que nadie cuestionase sus privilegios. Se trataba de comprender si empresas como la National Electric Light Association o la American Gas Association hubiesen interferido - y de qué manera- en el debate político relativo a una posible nacionalización de los servicios energéticos.

Una indagación análoga se había realizado entre 1925 y 1927. Los resultados, no obstante, habían sido decepcionantes, de ahí que algunos políticos hubiesen solicitado la institución de un comité ad hoc por parte del Senado $^{18}$. La propuesta generó un enfrentamiento entre quienes defendían la necesidad de acabar con los abusos empresariales y quienes consideraban que el sector estaba siendo sometido a un proceso inquisitorial por parte del poder político. El compromiso, al final, fue el establecimiento de una nueva investigación de la Federal Trade Commission ${ }^{19}$. Ignorada por gran parte de la ciudadanía durante sus fases iniciales, la labor de los investigadores adquirió enorme importancia tras la eclosión de la crisis, lo que ciertamente influyó en la decisión de prolongar las indagaciones durante seis años.

Demostrar la existencia de las actividades de propaganda no fue una tarea complicada, pues era del todo evidente que las corporaciones habían difundido imponentes campañas sobre la temática de la distribución de la energía ${ }^{20}$. Se descubrió que habían enviado material propagandístico a miles de periódicos ${ }^{21}$. Se encontraron evidencias de propaganda en los centros educativos ${ }^{22}$. Se investigaron las cartas que los ciudadanos habían recibido por correo ${ }^{23}$. Se examinó la relación entre determinadas produc-

18 «Authorizing an Investigation of Public Utility Corporations», S. Res. 83, $70 .^{\circ}$ congreso, 17 de diciembre de 1927.

19 Thompson, 1932, pp. XIX-XX. Creada en 1914 como una agencia independiente, el principal objetivo de la Federal Trade Commission es el de evitar la competencia desleal, de ahí que el Congreso le otorgase la autoridad de realizar investigaciones y poderes casijudiciales para castigar malas prácticas y abusos. Véase Holt, 1922, pp. 1, 17 y 27.

${ }^{20}$ Gruening, 1964, p. XI.

21 «6,500 Newspapers Receive Propaganda from Utility Bureau», Chicago Daily Tribune, 18 de mayo de 1928, p. 20.

22 «Utility Propaganda Work at Colleges Upheld at Hearing», The Washington Post, 7 de julio de 1928 , p. 7.

${ }^{23}$ «Asks Postal Inquiry in Farm Board Attack», The New York Times, 17 de octubre de 1930 , p. 41. 
toras cinematográficas y las empresas del sector ${ }^{24}$. Se habló de denegar las licencias radiofónicas a las emisoras que, a cambio de generosas subvenciones, habían denigrado durante sus programaciones la gestión pública de los servicios energéticos ${ }^{25}$. En su informe final, la Federal Trade Commission anunció haber descubierto una operación de propaganda que, con la excepción de la experiencia bélica del Committee on Public Information, por su extensión temporal y por lo extraordinarios medios empleados, no tenía precedentes en la historia de los Estados Unidos: «[They] have literally employed all forms of publicity except "sky writing", and frequently engaged in efforts to block full expression of opposing views (...) measured by quantity, extent, and cost, this was probably the greatest peace-time propaganda campaign ever conducted by private interests in this country» 26 .

La investigación fue cubierta con gran atención por la prensa, lo que indudablemente benefició a la nueva administración. Roosevelt se instaló en el 1600 de Pennsylvania Avenue en marzo de 1933. El país se encontraba todavía en una situación económica catastrófica y la Casa Blanca se preparaba para dar un viraje radical hacia el intervencionismo estatal en economía. Las noticias relativas a los abusos de las empresas facilitaron esta tarea. A mediados de los años treinta la administración lanzó un ataque frontal contra el sistema de poder de las grandes corporaciones. Más allá de las ya mencionadas Glass-Steagall Banking Act y Securities Exchange Act, en 1935 se aprobó la National Labor Relations Act, con la que se puso límite a las medidas antisindicales de los empleadores, creándose además la National Labor Relations Board.

En cuanto a las empresas que la Federal Trade Commission había estado investigando, en 1935 la Wheeler-Rayburn Act se propuso realizar una profunda regularización del sector eléctrico. Los efectos materiales de la Gran Depresión, por una parte, y las revelaciones de la Federal Trade Commission, por otra, habían creado un clima de opinión favorable a la restricción de las libertades de los grandes colosos económicos, y

24 Monticone, 2015.

25 A cientos de estaciones se les requirió revelar si habían recibido contribuciones por parte de los empresarios del sector. Véase NARA, RG122, Economic Investigations File, 1915-1938, Power \& Gas, caja 3707, Cor 5 Fed 6.

${ }^{26}$ Comisión Federal de Comercio, Utility Corporations, Summary Report of the Federal Trade Commission on Efforts by Associations and Agencies of Electric and Gas Utilities to Influence Public Opinion, Government Printing Office, Washington, 1934, p. 391. 
eso pese a que la cultura del laissez faire estaba muy arraigada en el país. Cuando Burton K. Wheeler habló, durante un programa radiofónico, de la necesidad de reducir el poder de las corporaciones de la energía, recibió numerosas cartas de apreciación por parte de los oyentes ${ }^{27}$. En el Congreso se planteó incluso la nacionalización del sector, una propuesta que acabó siendo conocida como la death sentence (literalmente, la condena a muerte de las corporaciones). No obstante, repentinamente fueron presentadas propuestas de ley bastante más blandas, como por ejemplo la que limitaba el campo de acción de las empresas a un único estado ${ }^{28}$.

Este repentino giro hacia la moderación decepcionó a una parte de los sostenedores del New Deal. Muchos de ellos comenzaron a sospechar que, una vez más, las grandes corporaciones estuviesen contaminando el proceso legislativo. ¿De verdad los colosos del sector se habían resignado a una legislación que podía destruir su negocio? ¿O estaban más bien interfiriendo en la labor de los representantes del pueblo? En el Congreso hubo quien consideró necesario que un nuevo comité legislativo investigase posibles actos improcedentes. Hugo Black — un obstinado partidario del New Deal- solicitó el establecimiento de una indagación en el Senado. John O'Connor - un crítico de las políticas intervencionistasharía lo mismo en la Cámara de los Representantes. Los mismos hechos finalmente serían investigados por comités distintos, dos investigaciones que acabarían siendo dominadas por la encendida rivalidad de personajes que encarnaban posturas ideológicas drásticamente contrapuestas.

\section{Los comités sobre las actividades de lobby: radicalización ideológica y deriva emocional}

El establecimiento de investigaciones legislativas sobre las campañas de presión contaba con destacados antecedentes. En 1913 el presidente Wilson había denunciado las actividades de lobby de las compañías que se oponían a la Tariff Act, lo que llevó a la institución de un subcomité de investigación en la Comisión de Justicia del Senado y de un comité especial en la Cámara de los Representantes ${ }^{29}$. Sin embargo, ninguna de las

27 NARA, RG46, Federal Power Trade Reports and Various Sub, caja 120, Sen 74AF12, Various Subjects (aug. 1935).

${ }^{28}$ Geddes, 1996, p. 63.

29 Perros, 1956, pp. 1-2. 
dos investigaciones finalmente aportó legislación que pudiese acabar con dichas prácticas. En 1929 Thaddeus H. Caraway presidió un subcomité de la Comisión de Justicia del Senado sobre supuestas actividades de presión en el Distrito de Columbia ${ }^{30}$. Una vez más, no fueron propuestas medidas concretas, aunque algunos periódicos señalaron que el comité, por lo menos, había despertado la atención de los electores sobre este fenómeno: «The Caraway lobby committee has been useful in teaching the people to look beneath the surface of lobbying organizations pretending to be working solely for the public welfare» ${ }^{31}$.

En 1935 se pusieron en marcha dos nuevas indagaciones, que desembocarían en una grave lucha institucional, abriendo además profundas heridas en el Partido Demócrata. El senador Black logró que se instituyera un comité especial sobre las actividades propagandísticas contra la ley Wheeler-Rayburn. Se trataba de un político conocido por su hostilidad hacia los grandes poderes económicos, teniendo además en su historial la mancha de haberse afiliado, desde 1922 a 1925, al Ku Klux Klan ${ }^{32}$. Poco después en la Comisión de Normas de la Cámara de los Representantes se abrió una investigación análoga. Su líder sería O'Connor, él también demócrata, quien había manifestado tener poco entusiasmo por el programa intervencionista de Roosevelt ${ }^{33}$. Los intentos de crear un comité conjunto fracasaron precisamente debido a su incompatibilidad ideológica. En la Cámara de los Representantes se investigarían sólo las actividades relativas a la Wheeler-Rayburn, mientras que en el senado se indagó todo tipo de actividades impropias de las empresas. El comité de O'Connor, por su parte, extendió su investigación también a las acciones propagandísticas de los funcionarios gubernamentales ${ }^{34}$. Black, en cambio, se centró sólo en las corporaciones, negándose por ejemplo a indagar - como le reque-

${ }^{30}$ Ledbetter, 2005, p. 141. Durante las audiencias Hiram Bingham fue censurado por haber beneficiado a la Manufactures' Association. El senador, célebre por su expedición al Machu Picchu, admitió haber contratado, con dinero público, a Charles L. Eyanson, un empleado de la Manufacturers' Association (Connecticut). Eyanson pudo asistir a los trabajos de la Comisión de Finanza del Senado, que estaba entonces debatiendo sobre aranceles. Véase «The Censure Case of Hiram Bingham of Connecticut (1929)», 2019.

31 «Propaganda Finances», The Washington Post, 13 enero de 1930, p. 6.

${ }^{32}$ La organización xenófoba había respaldado abiertamente, en Alabama, la campaña electoral que lo había llevado por primera vez al Congreso. Véase Mckenna, 2002.

33 Wolf, Pederson y Daynes, 2001, p. 64.

34 «House Votes Wide Inquiry on Lobbying», The Herald Tribune Bureau, 9 de julio de 1935 , p. 1A. 
ría una enmienda del republicano Daniel O. Hastings - el supuesto empleo de dinero público para la organización de las manifestaciones de los agricultores favorables al New Deal ${ }^{35}$.

Los comités acabaron enfrentándose entre sí ante el tribunal de la opinión pública. Hubo momentos altamente embarazosos. O'Connor no dudó en ridiculizar la labor de sus colegas del Senado: «[All they had done is to] rush in and beat us to it .... I don't think they have touched on much yet» ${ }^{36}$. Black llegó incluso a investigar al hermano de O'Connor - un prestigioso abogado que había trabajado también con el presidente Roosevelt- revelando que se había hecho pagar de la Gas \& Electric Co. - una de las empresas sospechosas de ejercer presiones sobre el Congreso- 25.000 dólares por una asesoría legal ${ }^{37}$. Hubo enfrentamientos también a la hora de hacerse con los testigos. Cuando Black intentó arrastrar ante su comité al empresario Howard C. Hopson, O’Connor intentó impedirlo ${ }^{38}$.

Entre las pruebas de las campañas de presión, Black presentó los 816 telegramas que el representante Denis J. Driscoll había recibido por parte de personas que declaraban oponerse a la Wheeler-Rayburn. El senador acusó a la Associated Gas and Electric Company de haber pagado a todos sus autores ${ }^{39}$. Su comité envió cuestionarios a decenas de empresas, exigiéndoles aclarar si habían financiado campañas contra dicha ley ${ }^{40}$. Black denunció que las actividades de presión no se detenían ni siquiera ante el comité del Senado que las estaba indagando, asegurando que él mismo había recibido numerosas cartas que lo invitaban a renunciar a la investigación. En su opinión, se trataba seguramente de una maquinación organizada por alguna corporación: «Quite a number of propaganda letters have come to me from Anniston in the last few days. They are written with the same typewriter, and many of them are identical, even as to punctuation ${ }^{41}$.

35 «All of House Asked to Aid Lobby Probe», The Hartford Courant, 12 de julio de 1935 , p. 8 .

${ }^{36}$ Citado en Gregory y Strickland, 1976, p. 552.

37 «Prober's Kin Paid \$25,000 by Big Utility: Roosevelt Ex-Partner», The Washington Post, 31 de julio de 1935, p. 1.

38 «Senate-House Rift Grows as O’Connor Holds Hopson», The New York Times, 15 de agosto de 1935, p. 1.

39 Van Der Veer Hamilton, 1982.

40 NARA, RG46, Special Committee on Lobbying, General Files, caja 1.

${ }^{41}$ Carta de Hugo Black al juez Charles S. Layden (23 de julio de 1935), NARA, RG46, Special Committee on Lobbying, General Files, caja 6. 
O'Connor reveló que numerosas empresas habían ejecutado una «extensa, bien organizada y bien financiada campaña» - que incluía telegramas, artículos, cartas, circulares, anuncios, etc. - consagrada a «prevenir la promulgación de legislación adversa». Añadió sin embargo que no se habían encontrado evidencias de «contactos impropios» con los legisladores: «All this may be called, in a broad sense, propaganda. But the term 'propaganda' when used invidiously connotes insidious and indirect publicity, wherein the individual and public opinion sought to be created is hidden. In this instance, there is no evidence of propaganda of this kind ${ }^{42}$. O'Connor, en suma, reconocía la existencia de actividades de presión, pero parecía legitimarlas. Entre los demócratas se difundió la sospecha de que su investigación no había tenido otra finalidad que la de proteger a las empresas. No fue probablemente ninguna coincidencia que perdiese su escaño en las siguientes elecciones.

Black, en cambio, se convirtió en un ejemplo a seguir para los partidarios más radicales del New Deal. El senador no dudó en trasformar su comité en un agente de lucha política. El New York Herald Tribune lo acusó de haber usado el comité como un arma al servicio del ideal intervencionista: «The Senate Lobby investigation is being forced into a weapon on which New Deal supporters evidently except to rely heavily during the coming year of political conflict» ${ }^{43}$. Philip H. Gadsden, director del Committee of Public Utility Executives, invitó a los congresistas a ignorar las revelaciones del comité: «The disclosures before that committee must not be utilized to create an atmosphere of passion and prejudice in which the sponsors of government ownership can force the passage of any bill, however destructive of the public interest» ${ }^{44}$.

Con el paso del tiempo la actuación de los investigadores se hizo cada vez más radical. Se llegó a pedir la detención de testigos que no habían acudido ante el comité, aunque sí se habían presentado ante el equipo de O'Connor ${ }^{45}$. Con la complicidad de Western Union y de la Federal Com-

42 Commisión de Normas de la Cámara de los Representantes: Investigation of Lobbying Activities, Report No.2081, Committee on Rules, House of Representatives, $74^{\text {th }}$ Congress, $2 d$ Session, 27 de febrero 1936, p. 2.

${ }^{43}$ Lindley, Ernest K., «Lobby Inquiry Seen Political Weapon in '36», New York Herald Tribune, 21 de julio de 1935, p. 14.

44 Declaración de Philip H. Gadsden, director del Committee of Public Utility Executives (22 de julio de 1935), NARA, RG46, Special Committee on Lobbying, General Files, caja 1.

45 Comité Especial del Senado sobre actividades de lobby: Investigation of Lobbying Activities, Partial Report (No. 1272) Senate, 74th Congress, 1st Session, 29 de julio 1935, p. 2. 
munications Commission, además, se consiguieron cientos de telegramas privados, entre ellos los de poderosos personajes como el magnate de la información William Randolph Hearst o el abogado Silas Strawn. Denunciado ante los tribunales de justicia, Black tuvo finalmente que renunciar a utilizar los telegramas como pruebas. El incidente manchó gravemente la imagen del comité, acabando sus métodos inquisitoriales incluso en el punto de mira de muchos demócratas ${ }^{46}$. En la prensa dichos métodos fueron denunciados como ajenos a la cultura democrática estadounidense. En el Chicago Tribune apareció una viñeta con una representación de Black que montaba a caballo con las vestimentas del Ku Klux Klan. En su mano llevaba una bandera en la que se leía «Black Inquisition». Una inscripción le acusaba de secuestrar telegramas privados e intimidar a todos los que se oponían a las políticas del New Deal ${ }^{47}$. Lo cierto es que el paso del tiempo no ha logrado borrar esta impresión. A lo largo de las décadas, han sido muchos quienes han seguido refiriéndose al senador sureño empleando la palabra «inquisidor». Recientemente, David T. Beito ha asegurado que Black puso en marcha una «vigilancia masiva» de los críticos del New Deal, espiando comunicaciones privadas a una escala nunca vista en época de paz: "A case can be made that the Black Committee posed a greater threat to individual rights than the "witch-hunts" of the 1940s or 1950s because it enjoyed comparatively vast powers of mass surveillance» ${ }^{48}$.

En 1937 Roosevelt nombró a Black como miembro de la Corte Suprema, una designación que obviamente levantó grandes polémicas. La dirección del comité sobre las actividades de lobby recayó entonces en el senador Sherman Minton. Muchos periódicos lo acusaron de perseverar en la persecución de los opositores políticos: «The only reason offered for summoning the publisher of Rural Progress to testify before the committee was that the magazine has carried articles deemed unjustly critical of the Administration ${ }^{49}$. Con todo, tras el abandono de Black el comité perdió paulatinamente la capacidad de atraer la atención de la opinión pública. Se convocaron cada vez menos audiencias, clausurándose sus actividades en 1938. Mientras tanto, la Wheeler-Rayburn Act había sido aprobada en su versión más moderada. Se había restringido considerablemente

\footnotetext{
${ }^{46}$ Hulnick, 2016, p. 70.

47 NARA, RG46, Special Committee on Lobbying, General Files, caja 1.

48 Beito, 2018, pp. 169-201.

49 «Broadening its Scope», The Washington Post, 8 de mayo de 1938, p. B8.
} 
el campo de acción de las empresas de la energía, y sin embargo la llamada death sentence no llegó a promulgarse ${ }^{50}$.

\section{La investigación sobre el negocio de guerra: entre realidad y teorías conspiratorias}

Durante los años treinta, los traumas socioeconómicos y psicológicos creados por la crisis, por una parte, y el deseo de protagonismo de determinados congresistas, por otra, llevaron al establecimiento de comités de investigación que explotaban sentimientos como el miedo, el rancor o la sensación de desprotección. Algunas de las investigaciones más mediáticas de la época estuvieron relacionadas con el mundo militar. En un momento de grave inestabilidad social, los intereses económicos de la industria de las armas y las relaciones entre los altos cargos del ejército y el universo financiero se convirtieron en temas muy atractivos para los congresistas.

Entre 1929 y 1930 un subcomité de la Comisión de Asuntos Navales del Senado investigó la contratación, por parte de las empresas de la construcción naval, de William B. Shearer, un propagandista al que se le había supuestamente encargado boicotear toda tentativa política de restringir el armamento de la marina ${ }^{51}$. En particular, se le acusaba de haber causado el fracaso de la Conferencia de Ginebra de 1927, cuyo fin había sido el de alcanzar un acuerdo internacional para limitar la construcción de destructores y submarinos. Según la revista The Nation, fueron muchos los testigos que confirmaron estas sospechas: «So pervasive and influential were his activities that he was hailed in the English and European press as having been "the man who broke up the conference", a title he himself modestly does not deny» 52 .

Desde 1933 a 1937 los demócratas John W. McCormack y Samuel Dickstein investigaron en la Cámara de los Representantes la propaganda que los simpatizantes de los nazis alemanes difundían en los Estados Uni-

${ }^{50}$ La ley se mantuvo en vigor hasta 2005, cuando fue derogada por el Congreso.

51 Subcomité de la Comisión de Asuntos Navales: Alleged Activities at the Geneva Conference, Hearings Before a Subcommittee of the Committee on Naval Affairs, United States Senate, Seventy-First Congress, First Session, Government Printing Office, Washington, 1930.

52 Allen, Robert S., «Mr. Shearer Likes a Big Navy», The Nation, 129, 3353, 9 de octubre de 1929, p. 379. 
$\operatorname{dos}^{53}$. Durante las primeras fases de sus investigaciones, siguieron la pista de una presunta conspiración - el llamado Business Plot - abocada a destruir las instituciones democráticas. Algunos altos cargos militares y distintos exponentes del mundo de los negocios supuestamente habían estado planeando un golpe de estado para derrocar a Roosevelt. La investigación arrancó a partir de las revelaciones del general Smedley Darlington Butler, quien declaró haberse enterado de la trama criminal al haber sido contactado por algunos conjurados. El comité sostuvo que la conspiración había sido real, sin embargo - a falta de evidencias concretas - finalmente nadie se planteó tomar medidas para castigar la supuesta traición ${ }^{54}$.

En 1934 también arrancó en el Senado una investigación, liderada por el republicano Gerald P. Nye - un aislacionista radical - sobre las supuestas maniobras realizadas, durante la época prebélica, por las industrias armamentísticas y por algunos grandes bancos con el fin de involucrar a los Estados Unidos en la Gran Guerra. La investigación se había originado tras la publicación, aquel mismo año, del libro Merchants of Death ${ }^{55}$. La sospecha era que, durante los dos años previos a la intervención estadounidense de 1917, un consorcio de militares, hombres de negocios y periodistas hubiese plasmado en el país un clima de hostilidad permanente contra Alemania, obligando de facto a Wilson a intervenir en el conflicto. La postura del político republicano, al igual que la publicación de Merchants of Death, tiene que ser contextualizada en un periodo histórico - comenzado enseguida después del fin de la Primera Guerra Mundial - caracterizado por el auge de fuertes corrientes antimilitaristas. Muchos ciudadanos habían estado escribiéndoles a sus representantes pidiendo que se investigase la propaganda de la industria armamentística: «The undersigned citizens of Warkick (...) believing that war grows out of unreasonable distrust and fear which is fostered and promoted in the minds of people by carefully planned propaganda (...) request the Congress of the United States to investigate the propaganda methods of the munitions companies» ${ }^{56}$.

${ }^{53}$ Lo hicieron a través de los comités Nazi Propaganda Activities by Aliens in the United States (subcomité de la Comisión de Inmigración y Naturalización) y Special Committee on Un-American Activities on Nazi Propaganda. Véase Migliucci, 2021.

54 Marshall, 2008, p. 185.

55 Engelbrecht y Hanighen, 1934.

${ }^{56}$ Carta a Hamilton Fish por parte de ciudadanos de Warwick, Orange County, State of New York (28 de agosto de 1934), NARA, RG233, Petitions and Memorials, caja 398, HR 73A-H10.5 H10.6 H10.7. 
Cuando Nye abrió su investigación, una parte importante de la opinión pública le apoyó con entusiasmo. En los archivos del comité se encuentran decenas de cartas y telegramas que ciudadanos, centros religiosos y asociaciones cívicas enviaron con el fin de respaldar a los investigadores ${ }^{57}$. Un ciudadano recordó que, durante el periodo bélico, había estado a punto de acabar en la cárcel por decir lo que ahora el comité estaba postulando abiertamente en el Congreso ${ }^{58}$. Nye recibió muestras de apoyo incluso de asociaciones pacifistas en el extranjero ${ }^{59}$. En cuanto a la prensa, también fueron muchos los periódicos que se mostraron agradecidos: «As peoples become more awake to the revolting way in which arms profiteers have taken advantage of their patriotism they will surely demand honest and thorough government investigation similar to this now being undertaken by the Nye Committee [...] the most notable investigation yet made into the private armaments business» ${ }^{60}$.

En el Congreso, la causa de Nye fue abrazada por exponentes de distintos colores políticos. El mismo presidente Roosevelt le envió un mensaje de respaldo ${ }^{61}$. Muchos autores atribuyen a las presiones del político republicano las leyes sobre neutralidad que fueron aprobadas en la segunda mitad de los años treinta ${ }^{62}$. El demócrata Louis Ludlow, además, intentó que se promulgase una ley que preveía la institución de un referéndum popular para validar cualquier declaración de guerra que se tomase a nivel institucional. Su compañero de partido Fred J. Sisson confesó que él mismo había sido una de las víctimas de la formidable campaña propagandística de la época bélica: «If I had been in this Congress in 1917 I should unquestionably have voted for the declaration of war because I was carried away by that same hysteria which propagandists like Hearst and the paid agents of the profiteers inject into us» ${ }^{63}$. Cabe además recordar que la institución de un comité sobre la propaganda de las industrias

57 NARA, RG46, Special Committee Investigating the Munitions Industry, Exective File, cajas 175 y 215.

58 Carta de Charles Kroll al senador Gerald P. Nye (26 de diciembre de 1936), NARA, RG46, Special Committee Investigating the Munitions Industry, Exective File, caja 175.

${ }_{59}$ NARA, RG46, Special Committee Investigating the Munitions Industry, Exective File, caja 216.

60 «Patriotism or Profits?», The Christian Science Monitor, 5 de diciembre de 1934, pg. WM5.

61 J., «Arms Manufacturers and the Public», Foreign Affairs, 12, julio de 1934, p. 639.

62 Cole, 1962, p. 106. En particular, las Neutrality Act de 1935, 1936, 1937 y 1939.

${ }^{63}$ Congressional Record-House, 5 de abril de 1935, p. 5159. 
armamentísticas ya había sido solicitada en 1919 por el republicano Ernest Lundeen ${ }^{64}$.

Cuando a mediados de los años treinta la temática volvió de actualidad, Nye fue el político que más luchó para que se estableciese una investigación. Pese a que en el Congreso había una clara mayoría demócrata, el senador republicano logró hacerse con el cargo de chairman del comité. Se realizaron casi cien audiencias, a las que fueron llamados a declarar más de doscientos testigos. Nye pudo contar con la colaboración del Departamento de Justicia y del Departamento de Estado ${ }^{65}$. Este último permitió que se analizase la documentación relativa al hundimiento del RMS Lusitania ${ }^{66}$. El republicano tuvo menos suerte con la Biblioteca del Congreso, ya que ésta se negó a entregarle los diarios de Robert Lansing. Antes de su muerte, el ex Secretario de Estado los había donado a la institución cultural con la condición de que no se hiciesen públicos hasta 1949. El político se enfrascó en una larga polémica con el Bibliotecario del Congreso Herbert Putnam, quien se mantuvo firme en hacer respetar la voluntad del político ${ }^{67}$.

Nye intentó demostrar que, tras el estallido de la guerra en Europa en 1914 , los bancos y las industrias de las municiones habían intentado destruir la política de neutralidad de Wilson ejerciendo una gran presión sobre congresistas y miembros del Gobierno, y manipulando a la opinión pública con la complicidad de la prensa ${ }^{68}$. De esta manera, el mundo financiero habría protegido sus inversiones en el Reino Unido (que hubiesen peligrado en caso de victoria alemana). Las empresas de las armas, por su parte, habrían alentado la entrada en la guerra de los Estados Unidos con la mera finalidad de multiplicar sus ventas. Nye intentó remontarse a las razones por las que el Gobierno había ido aumentando la finan-

64 «Resolution to Investigate Foreign Propaganda in the United States», H. Res. 615, 65. ${ }^{\circ}$ Congreso, 28 de febrero de 1919.

65 En cuanto al Departamento de Justicia, véase NARA, RG60, D. J. Central Straight Numerical Files, caja 4326, 235636-235654.

66 Carta del secretario de Estado Cordell Hull al Fiscal General Homer Cummings (22 de marzo 1935), NARA, RG60, D. J. Central Straight Numerical Files, caja 4326, 235644. Se trata del transatlántico británico hundido por un sumergible alemán en mayo de 1915. Al haber habido un enorme número de estadounidenses entre las víctimas, el desastre generó en los Estados Unidos un fuerte sentimiento de hostilidad hacia los alemanes.

${ }^{67}$ NARA, RG46, Special Committee Investigating the Munitions Industry, Exective File, cajas 156 y 157.

68 «Arms Industry Is Found Guilty by Nye Report», The Christian Science Monitor, 20 de abril de 1936, p. 1. 
ciación destinada al ejército: «What do the munitions makers know about the propaganda behind the moves for larger appropriations for National Defense? ${ }^{69}$ En un discurso radiofónico, el republicano alertó sobre la enorme capacidad de manipulación mediática del sector: «Americans left to their good sense and Judgment will declare that never again will our country engage in war away from home. But never at any time is there let-up of that propaganda intended to convince us that other nations are more adequately prepared for war than are we» ${ }^{70}$.

De todas maneras, finalmente fueron bastante escasas las pruebas que el comité pudo presentar a sus compañeros del Congreso. Las acusaciones se hacían cada vez más escandalosas, y sin embargo las evidencias del complot destacaban por su ausencia. La administración de Roosevelt retiró paulatinamente su respaldo a la investigación. Newton D. Baker - secretario de la Guerra en la época en la que se habrían originado las manipulacionesaseguró que nadie había realizado presiones sobre el Gobierno con el fin de entrar en la guerra ${ }^{71}$. El Congreso fue reduciendo los fondos que el comité tenía a su disposición. En febrero de 1936 la indagación fue clausurada sin que se hubiese alcanzado ningún objetivo destacado. La Segunda Guerra mundial, además, acabó definitivamente con los sentimientos pacifistas. En marzo de 1941 la llamada Lend-Lease Act borró de un plumazo toda la política de neutralidad de la década de los treinta, permitiéndose la entrega de material bélico a las naciones aliadas. La batalla de Nye acabó cayendo en el olvido. Su labor fue ignorada durante mucho tiempo incluso por el mundo académico, condenándose durante décadas - tal y como afirmó Gianfranco Peroncini- a una damnatio memoriae historiográfica ${ }^{72}$.

\section{Propaganda en la gran pantalla: la última breve investigación antes de Pearl Harbor}

El fracaso de la investigación sobre la industria armamentística no desanimó a Nye. El político encontró pronto un nuevo camino para poder

69 Albright, Robert C., «Arms Makers of U.S. Foster War, Says Nye», The Washington Post, 2 de septiembre de 1934, p. M3.

${ }^{70}$ Congressional Record-Senate, 12 de abril 1934, p. 6458.

71 Baker, Newton D., «Why We Went to War», Foreign Affairs, 15 (1), octubre de 1936, p. 1.

72 Peroncini, 2014, p. 567. 
volver a librar su batalla aislacionista. Su nuevo objetivo fueron las major cinematográficas, a las que acusó de estar orquestando una colosal operación de propaganda para convencer a la opinión pública y al Gobierno de la necesidad de que los Estados Unidos le declarasen la guerra a la Alemania nazi. No se trataba de acusaciones completamente nuevas. En un informe que la Inteligencia Militar redactó en 1937, por ejemplo, se denunciaba la existencia de una Hollywood Anti-Nazi League ${ }^{73}$. Sospechas y rumores se multiplicaron tras el estallido, en septiembre de 1939, de la Segunda Guerra Mundial. Muchos políticos de la oposición plantearon la posibilidad de una alianza entre el mundo del cine y la Casa Blanca. En 1940 el representante Jacob Thorkelson denunció que la administración de Roosevelt estaba realizando una campaña de manipulación con el fin de convencer a la ciudadanía de la inevitabilidad de un conflicto con el régimen de Adolf Hitler: «I grant that the Administration has been able to organize a large and expensive war propaganda machine to confuse the public and to bring about fear of invasion and war ${ }^{74}$.

El 1 de agosto de 1941 Nye presentó, junto con el senador demócrata Bennett Champ Clark, una resolución con la que se solicitaba el establecimiento en la Comisión de Comercio Interestatal de un comité que indagara la propaganda intervencionista de Hollywood: «A complete investigation of any propaganda disseminated by motion pictures and radio or any other activity of the motion-picture industry to influence public sentiment in the direction of participation by the United States in the present European war» ${ }^{75}$. Clark se hizo con el cargo de chairman, aunque fue Nye el verdadero líder de la investigación. Otro miembro del comité fue el senador Wheeler, que, como vimos anteriormente, años antes había protagonizado una vigorosa batalla contra las empresas de la energía. La investigación, en efecto, tiene que ser interpretada como una expresión de los ideales aislacionistas, pero también - tal y como ha señalado John E. Moses - como el reflejo de aquel movimiento progresista que, desde ha-

73 «Communist activities in the motion picture industry», informe de la Inteligencia Militar (21 de agosto de 1937), NARA, RG165, Military Intelligence Division Correspondence, 1917-41, caja 2851, 10110-2666.

74 Appendix to the Congressional Record (A Pilot's Advice, Remarks of Hon. J. Thorkelson of Montana in the House of Representatives), 17 de junio de 1940, p. 3991.

75 «A Resolution Authorizing an Investigation of War Propaganda Disseminated by the Motion Picture Industry and of any Monopoly in the Production, Distribution, or Exhibition of Motion Pictures», S. Res. 152, 77. . congreso, 1 de agosto de 1941. 
cía ya muchos años, luchaba contra las grandes corporaciones ${ }^{76}$. Un papel destacado lo jugó también el grupo de presión - de tendencia ultraderechista- America First Committee y más en particular el periodista anticomunista John T. Flynn ${ }^{77}$. El comité recibió además el respaldo de personajes tan controvertidos como el populista Charles Coughlin - un sacerdote católico que en aquellos años exaltaba a las audiencias radiofónicas con discursos ideológicos anticapitalistas e incluso antisemitas - y el célebre aviador Charles Lindbergh, a quien se le atribuían actitudes filo-alemanas. Las productoras, por su parte, fueron defendidas nada menos que por Wendell Willkie, quien había sido el candidato republicano a la presidencia en las elecciones de $1940^{78}$.

El comité tuvo una existencia efímera y, sin embargo, conquistó la atención de los ciudadanos rápidamente, eso debido sobre todo a que fueron muchas las estrellas de Hollywood - por ejemplo Charles Chaplinque fueron llamados a declarar ${ }^{79}$. Las audiencias tuvieron lugar entre el 9 y el 26 de septiembre de $1941^{80}$. Para demostrar la teoría de la conspiración cinematográfica, Nye señaló ocho películas que las grandes industrias del cine habían supuestamente lanzando al mercado con el fin de convertir a los espectadores a la causa de la guerra ${ }^{81}$. En más de una ocasión se señaló que muchos de los líderes del imperio cinematográfico eran judíos, considerándose este hecho como una evidencia que - por sí sola - demostraría la existencia de una conspiración contra los nazis ${ }^{82}$. Desde el primer momento, numerosos periódicos valoraron los argumentos de Nye como extremadamente convincentes. En el Chicago Daily Tri-

76 Moser, 2001, p. 733.

77 Birdwell, 1996, p. 216.

78 «Willkie Will Represent Film Industry at Hearing», Los Angeles Times, 2 de septiembre de 1941, p. 18.

79 «Chaplin Called as Witness in Senate Film Investigation», Los Angeles Times, 14 de septiembre de 1941.

80 Subcomité de la Comisión de Comercio Interestatal del Senado: Propaganda in Motion-Pictures: Hearing before a Subcommittee of the Committee on Interstate and Foreign Commerce, United States Senate, Seventy-Seventh Congress, First Session, Government Printing Office, Washington, 1942.

81 «Eight Films Listed by Nye as Part of 'War Propaganda'», The Christian Science Monitor, 9 de septiembre de 1941, p. 8. Se trataba de The Great Dictator (Charlie Chaplin: 1940); Convoy (Pen Tennyson: 1940); Flight Command (Frank Borzage: 1940); Escape (Mervyn LeRoy: 1940); I married a Nazi (Irving Pichel: 1940); That Hamilton Woman (Alexander Korda: 1941); Man Hunt (Fritz Lang: 1941); y Sergeant York (Howard Hawks: 1941).

82 Birdwell, 1996, p. 317. 
bune apareció un artículo titulado The Enemy Within, en el que se acusaba a la industria del cine de haber creado una «war cospiracy» ${ }^{83}$. En algunas ciudades se llegaron a organizar protestas - incluso un boicot - contra los teatros que trasmitían las películas que venían mencionadas durante las audiencias ${ }^{84}$.

La investigación se interrumpió abruptamente en diciembre, debido al ataque japonés contra la base naval de Pearl Harbor ${ }^{85}$. El comité se disolvió - «en el interés de la unidad nacional»- poco después de la entrada en la guerra de los Estados Unidos ${ }^{86}$. La labor del equipo de Nye puede ser considerada como un claro antecedente del proceso político y mediático en contra de Hollywood que tendría lugar (esta vez por las supuestas enseñanzas comunistas de las películas) en la posguerra, durante los años del macartismo. El 10 de septiembre de 1941, en un artículo que el Washington Post dedicó a la investigación de Nye, se pudo leer la expresión «caza de brujas» ${ }^{87}$. Lo cierto es que Nye defendía que las películas eran mero entretenimiento, de ahí que les negase el derecho a la libertad de expresión previsto por el Bill of Rights ${ }^{88}$.

A distancia de años, varios historiadores han señalado que no era ningún disparate la teoría según la cual las grandes productoras intentasen persuadir a la opinión pública de la necesidad de acabar con los nazis. Según Michael Birdwell, si el comité hubiese tenido más tiempo y hubiese secuestrado material en los grandes estudios, habría ciertamente conseguido las pruebas de que Warner Bros. y las demás corporaciones habían estado respaldando la campaña por la intervención ${ }^{89}$. De ser ese el caso, de todas maneras, cabe preguntarse si una movilización de este tipo podría haber sido tachada de ilegal. Si la guerra no la hubiese interrumpido, lo más probable es que, tarde o temprano, la investigación sobre el mundo del cine habría llegado, igual que todos los comités que la habían prece-

83 «The Enemy Within», Chicago Daily Tribune, 4 de agosto de 1941, p. 12.

84 «Theaters Using Pictures on War Face a Boycott», Chicago Daily Tribune, 20 de septiembre de 1941, p. 8.

85 Sbardellati, 2008, pp. 412-436.

86 «Senate Drops Film Inquiry: Investigation of War Propaganda Tabled in Interest of Unity», Los Angeles Times, 6 de enero de 1942, p. 11.

87 Nover, «On Propaganda: Witch Hunt on Capitol Hill», The Washington Post, $10 \mathrm{de}$ septiembre de 1941, p. 11.

${ }^{88}$ Canham, Erwin D., «Down the Middle of the Road: Freedom of Film Speech», The Christian Science Monitor, 12 de septiembre de 1941, p. 22.

89 Birdwell, 1996, p. 368. 
dido, a un punto muerto. Los congresistas aseguraban que la propaganda constituía el cáncer del sistema democrático. Y, sin embargo, era precisamente la naturaleza liberal del régimen democrático lo que permitía la existencia de un gran número de campañas propagandísticas. Contrariamente a lo que ocurría en Alemania o en la Unión Soviética, en efecto, en el sistema político estadounidense los ciudadanos y las empresas tenían la posibilidad de producir las campañas mediáticas que consideraban más beneficiosas para sus intereses.

\section{La difusión de propaganda: ¿un delito o una manifestación de la libertad de expresión?}

El denominador común de todos los comités que investigaron la propaganda de las empresas privadas durante el periodo que ha sido objeto de estudio es que finalmente sus resultados fueron bastante decepcionantes. La causa de sus fracasos no solía ser la inconsistencia de las pruebas. Más allá de algunas teorías conspirativas y de unas cuantas paranoias complotistas, los problemas analizados por los comités eran reales, presentándose frecuentemente evidencias que demostraban la existencia de campañas propagandísticas y de actividades de presión. La verdadera cuestión era el hecho de que raramente dichas actividades violaban la legislación de la época. Los comités denunciaban una y otra vez las campañas de las empresas, resultándoles sin embargo imposible poner en marcha iniciativas de contención y represión. Durante la investigación sobre la propaganda de las productoras cinematográficas, se le preguntó a Nye si existía la posibilidad de que su comité llegase a proponer algún tipo de ley para hacer frente a la amenaza. El senador tuvo que admitir que dicha posibilidad ni siquiera se contemplaba ${ }^{90}$. Unos años antes, la Chattanooga Power Public League le había preguntado a la Federal Trade Commission la razón por la que la agencia no ponía fin a los abusos de las empresas: «It seems to us that it would be more important to stop such practices than to investigate them after they have been accomplished» ${ }^{91}$. Desde el Distrito de Columbia se contestó que, simplemente, la agencia no tenía jurisdicción para

90 «No Legal Basis», Los Angeles Times, 10 de septiembre de 1941, p. A4.

91 Carta de Hays Clark (Chattanooga Public Power League) a la Federal Trade Commission (18 de febrero de 1934), NARA, RG122, Economic Investigations File, 19151938, Power \& Gas, caja 3692, Cos 5 Cha 87. 
ello $^{92}$. Lo cierto es que, tras desvelar las maquinaciones propagandísticas de las grandes corporaciones, los comités se encontraban a menudo en la embarazosa situación de no poder proponer medidas legislativas que pudiesen castigar los comportamientos que habían denunciado como impro$\operatorname{pios}^{93}$.

Cabe además recordar que las actividades de propaganda y de presión no sólo no eran consideradas ilegales por la legislación de la época, sino que - al ser interpretadas como muestras de la libertad de expresión y de la defensa del mercado sin trabas - formaban parte de la identidad colectiva de los estadounidenses. Lo cierto es que en aquellos años muchos empresarios y numerosos electores reivindicaron su derecho, como ciudadanos de la República, de poder oponerse, con todos los medios a su alcance, contra cualquier tipo de legislación que limitase la libertad empresarial: «[The Federal Trade Commission] seem to have established opposition to public ownership as a crime (...) I also assert my right to oppose public ownership on the platform; in the press; in private conversation; in the voting booth and everywhere else; including schools» ${ }^{94}$. Desde las páginas de su periódico The United States News, el célebre periodista conservador David Lawrence reivindicaba con orgullo la organización de campañas de opinión en contra de la legislación intervencionista: «It is not propaganda or lobbying to rent advertising space or lease time over the radio. It is not propaganda or lobbying to ask those citizens of the country who concur in the sentiments expressed in a public appeal to send their communications and petitions to the members of Congress through the United States mails» ${ }^{95}$.

92 Carta de Ewin L. Davis (Federal Trade Commission) a Hays Clark (Chattanooga Public Power League) (18 de febrero de 1934), NARA, RG122, Economic Investigations File, 1915-1938, Power \& Gas, caja 3692, Cos 5 Cha 87.

${ }_{93}$ Las únicas excepciones fueron, ya en vísperas de la Segunda Guerra Mundial, aquellas leyes que perseguían la propaganda de ciudadanos, empresas o gobiernos extranjeros, por ejemplo la Foreign Agents Registration Act (1938), la Voorhis Act (1939), y la Alien Registration Act (1940).

94 Carta de H. B. Creel a William E. Humphrey, de la Federal Trade Commission (23 de junio de 1928), NARA, RG122, Economic Investigations File, 1915-1938, Power \& Gas, caja 3699, Cor 5 Cre 5.

95 El artículo de David Lawrence, titulado «Propaganda» y publicado en el United States News (sin fecha, ¿1935?), se encuentra en los archivos del Senado. Véase NARA, RG46, Papers Relating to Specific Bills and Resolutions, Sen 74A-E1, (Propaganda), caja 57, S.1725. 
En 1935 la Corte Suprema declaró ilegales algunas importantes reformas del New Deal, unas sentencias que, sin duda, animaron a quienes consideraban que el intervencionismo de Roosevelt y de sus seguidores era profundamente antiamericano. El Washington Post les llegó a sugerir a las corporaciones de gas y electricidad que admitiesen sin miedo, ante el comité de Black, haber luchado contra una legislación que ponía en peligro sus negocios: «All the utility fellows had to say to make the committee look ridiculous was: "Gentlemen of the committee, you can bet your boots we spent every nickel we could rake and scrape and did everything under the sun to defeat this bill. We must admit that we are so perverse that when a government tries to destroy us we get panicky and try to prevent it doing so"»»" .

Además, cuando intentaron demostrar la existencia de verdaderos delitos (por ejemplo el golpe de estado contra Roosevelt), los investigadores nunca pudieron aportar las pruebas necesarias. La investigación sobre los mercaderes de muerte es un buen ejemplo de ello. De acuerdo con Matthew Coulter, Nye no pudo probar que la industria armamentística había provocado la intervención estadounidense en la guerra ${ }^{97}$. Entre los documentos de su comité se encuentra un estudio relativo a las razones por las que 69 periódicos del Middle West dieron un giro, entre 1916 y 1917, hacia el intervencionismo. En sus conclusiones se leía que no existían pruebas de que hubiesen sido sobornados por la industria armamentística: «I doubt seriously that any of these newspapers were bought but, if they were bought, the purchaser did not get much for his money» ${ }^{98}$. En el informe final del comité se tuvo que admitir que no había sido posible demostrar que la intervención en el conflicto se había producido debido a las actividades de los fabricantes de $\operatorname{armas}^{99}$.

Ante la imposibilidad de poder demostrar la perpetración de delitos, los investigadores se conformaron con denunciar de forma reiterada aquellos actos y comportamientos que podían provocar rechazo en un determinado sector de la opinión pública, lo que sin duda favorecería la causa

96 «Along the Potomac», The Washington Post, 17 de julio de 1935, p. 7.

97 Coulter, 1997.

98 «The War Cry», NARA, RG46, Special Committee Investigating the Munitions Industry, Exective File, caja 158.

99 Comité especial de investigación del Senado sobre la industria de las armas: Report of the Special Committee on Investigation of the Munitions Industry, U.S. Congress, Senate, $74 .^{\circ}$ congreso, 24 de febrero de 1936. 
política que cada uno de ellos estaba defendiendo. No es de extrañar que, con el tiempo, las relaciones con la prensa llegasen a convertirse en una obsesión para los investigadores. Cuando el periodista Paul Wootan solicitó poder tomar fotos durante las audiencias de la investigación sobre la propaganda de las corporaciones de la energía, desde la Federal Trade Commission se le contestó que no sólo se le concedía el permiso, sino que se suspenderían los trabajos del comité durante unos minutos para que el periodista pudiese realizar su labor de la mejor manera posible ${ }^{100}$. Políticos como Nye llevaron las actividades de comunicación pública a otro nivel, denunciando constantemente la presencia de actores perversos - los bancos, las industrias de las armas, las corporaciones cinematográficas, etc. - que conspiraban en contra del pueblo. El republicano había entendido perfectamente que, cuanto más escandalosas eran sus revelaciones, más grande era el espacio que la prensa acabaría concediéndole.

A largo plazo, sin embargo, ciertas posturas y determinados métodos de los investigadores acabaron creando rechazo. Como hemos podido comprobar, la respuesta de las instituciones al desafío de la manipulación de la opinión pública por parte de las empresas con el tiempo fue politizándose. Las dos investigaciones que se establecieron en 1935 - ambas relativas a las actividades de presión de las empresas de la energía - son el ejemplo fehaciente de la deriva ideológica que tomaron, desde mediados de los años treinta, los comités legislativos de investigación. El empleo de técnicas inquisitoriales - por ejemplo el secuestro de correspondencia privada por parte de Black - hizo el resto. A largo plazo también causaron recelo los excesos mediáticos de Nye. El político republicano llegó a acusar de complicidad con las empresas armamentísticas al difunto presidente Wilson, lo que le costó la reprobación de una parte importante del mundo político y de la sociedad ${ }^{101}$. Un autor de la época señaló que Nye desapareció de la escena política con la misma rapidez con la que había adquirido popularidad: «Nye's career has been much like that of a rocket -a rapid rise accompanied by same bursts of light that give promise of what is to come, then a brilliant display followed by a burning descent» 102 .

100 NARA, RG122, Economic Investigations File 1915-1938, Power \& Gas, caja 3726, COR McG7.

101 «Nye Denounced as Coward for Wilson Attack», Chicago Daily Tribune, 18 de enero de 1936, p. 1.

102 Sayre, 1946, p. 127. 


\section{Conclusión}

A lo largo de este trabajo hemos visto como, durante los años de la Gran Depresión, en el Congreso de los Estados Unidos fueron establecidos numerosos comités de investigación para examinar las actividades de lobby y las campañas propagandísticas de distintos tipos de empresas. Muchos de ellos alcanzaron enormes cotas de popularidad y, sin embargo, en un determinado momento su influjo sobre las masas se extinguió repentinamente. El hartazgo de los ciudadanos ante la repetición obsesiva de determinadas acusaciones - sin que éstas se viesen acompañadas por iniciativas concretas que pudiesen solucionar el problema de raíz - llevaba a la inevitable clausura de los comités. Sin embargo, la persistencia del clima de desconfianza hacia el mundo empresarial y el perdurar de condiciones socioeconómicas extremadamente precarias llevaban puntualmente al establecimiento de nuevos comités consagrados a investigar otros potenciales abusos. Este ciclo se repitió una y otra vez, desde el Jueves Negro hasta Pearl Harbor.

Toda la problemática de la propaganda de las empresas giraba alrededor de dos cuestiones, una jurídica y otra conceptual. ¿Eran dichas campañas el espejo de un abuso de las clases privilegiadas o una muestra de resistencia del ciudadano ante el estado opresor? Y, sobre todo, más allá de lo que cada uno opinaba en relación con su legitimidad, ¿existían los extremos para señalarlas como delito? A lo largo de toda la década, las opiniones relativas a su integridad fueron heterogéneas, siendo en cambio constante la dificultad de los comités a la hora de poder demostrar que una campaña de propaganda había quebrantado la ley. Al fin y al cabo el entero ordenamiento jurídico de los Estados Unidos defendía como valores sagrados la libertad de empresa y la libertad de expresión.

Fue sólo con la entrada de los Estados Unidos en la Segunda Guerra Mundial que se puso fin - por lo menos durante el periodo bélico- al largo ciclo de los comités de investigación sobre la propaganda de las empresas. Con dos frentes abiertos en Europa y en el Pacífico, la nueva prioridad de los políticos de todos los partidos fue la búsqueda de unidad. Ya no se trataba de investigar a las empresas, ni de reducir su poder. Se trataba más bien de convencerlas para que pusiesen todo su potencial al servicio del esfuerzo bélico. En este sentido, el caso de las productoras cinematográficas es extremadamente significativo. Tras el ataque japonés en las Hawái se les pidió que contribuyeran a moldear el espíritu patriótico 
de la ciudadanía ${ }^{103}$. Todos parecían haber olvidado que, sólo pocas semanas antes, se les había investigado en el Congreso con la gravísima acusación de estar manipulando a la opinión pública nacional.

\section{Fuentes}

Hathitrust Digital Library (base de datos), Biblioteca del Congreso, Washington D.C., Estados Unidos.

Proquest Congressional (base de datos), Biblioteca del Congreso, Washington D.C., Estados Unidos.

ProQuest Historical Newspapers (base de datos), Biblioteca del Congreso, Washington D.C., Estados Unidos.

RG46 (Records of the U.S. Senate), National Archives and Records Administration (sede de Washington D.C.), Committee on Interstate Commerce.

RG46 (Records of the U.S. Senate), National Archives and Records Administration (sede de Washington D.C.), Special Committee on Lobbying.

RG46 (Records of the U.S. Senate), National Archives and Records Administration (sede de Washington D.C.), Special Committee Investigating the Munitions Industry.

RG46 (Records of the U.S. Senate), National Archives and Records Administration (sede de Washington D.C.), Papers Relating to Specific Bills and Resolutions.

RG60 (General Records of the Department of Justice), National Archives and Records Administration (sede de College Park), D. J. Central Straight Numerical Files.

RG122 (Records of the Federal Trade Commission), National Archives and Records Administration (sede de College Park), Economic Division.

RG165 (Records of the War Department General and Special Staffs), National Archives and Records Administration (sede de College Park), Military Intelligence Division Correspondence.

RG233 (Records of the United States House of Representatives), National Archives and Records Administration (sede de Washington D.C.), Petitions and Memorials.

103 En 1942 la Office of War Information llegaría a publicar el Government Information Manual for the Motion Picture Industry, con las directrices gubernamentales para las corporaciones cinematográficas: «The purpose of this office is to assist the motion picture industry in its endeavor to inform the American people, via the screen, of the many problems attendant on the war program». Véase Oficina de Informacion de Guerra (películas), Government Information Manual for the Motion Picture Industry, Washington, D.C., Office of War Information, 1942. 
La lucha institucional contra la propaganda de las grandes empresas estadounidenses ...

\section{Referencias bibliográficas}

«The Censure Case of Hiram Bingham of Connecticut (1929)», US Senate, https://www.senate.gov/artandhistory/history/common/censure cases/112HiramBingham.htm, consultada el 30 de enero de 2020.

BeITo, David T., «New Deal Mass Surveillance: The "Black Inquisition Committee” 1935-1936», Journal of Policy History, 30, 2, 2018, pp. 169-201.

BIRDwell, Michael Edward, Celluloid soldiers: Warner Bros., Alvin C. York, and the Coming of World War II, Tesis doctoral, The University of Tennessee, 1996.

CoBb, Frank I., The Press and Public Opinion (11 diciembre 1919), Hathitrust Digital Library, https://babel.hathitrust.org/cgi/pt?id=uc2.ark:/13960/ t8x923v2n, consultada el 5 de agosto de 2017 desde la Biblioteca del Congreso.

Cole, Wayne S., Senator Gerald P. Nye and American Foreign Relations, Minneapolis, The University of Minnesota Press, 1962.

Coulter, Matthew Ware, The Senate Munitions Inquiry of the 1930s: Beyond the Merchants of Death, Westport, Connecticut, London, Greenwood Press, 1997.

CREEL, George, How We Advertised America: The First Telling of the Amazing Story of the Committee on Public Information that Carried the Gospel of Americanism to Every Corner of the Globe, New York-London, Harper \& Brothers, 1920.

CudAhy, Richard D. y Henderson, William D., «From Insull to Enron: Corporate $(\mathrm{Re})$ Regulation after the Rise and Fall of Two Energy Icons», Energy Law Journal, 26, 1, 2005, pp. 35-110.

Derbyshire, Jeff, Full Disclosure: The Pecora Commission and the Political Fight for Financial Reform, Tesis, Universidad de Vanderbilt, Nashville Tennessee, 2012.

ENGELBRECHT, Helmuth Carol y HANIGHEN, Frank Cleary, Merchants of Death, A Study of the International Armament Industry, London, Routledge \& Sons, 1934.

GEDDES, R. Richard, «Time to Repeal the Public Utility Holding Company Act», Cato Journal, 16, 1, primavera/verano 1996, pp. 63-76.

Gregory, William A. y STRICKLAND, Rennard, «Hugo Black’s Congressional Investigation of Lobbying and the Public Utility Holding Company Act: A Historical View of the Power Trust, New Deal Politics, and Regulatory Propaganda», Okla. L. Rev., 29, 1976, pp. 543-576.

Gruening, Ernest, The Public Pays... and still Pays. A study of Power Propaganda, New York, Vanguard Press, 1964.

Holt, Stull W., The Federal Trade Commission: Its History, Activities and Organization, New York, D. Appleton and Company, 1922. 
HulNiCK, Blake B., «Consumer Crusade: Justice Hugo Black as Senate Investigator», Journal of Southern Legal History, 24, 2016, pp. 69-108.

Johnson, Charles Thomas, Culture at Twilight: The National German-American Alliance, 1901-1918, New York, Peter Lang, 1999.

LedbetTer, Calvin R. Jr., «The other Caraway: Senator Thaddeus H. Caraway», The Arkansas Historical Quarterly, 64, 2, verano de 2005, pp. 123-145.

Marshall, Emily Lacy, The Forgotten Treason: The Plot to Overthrow FDR, Tesis doctoral, Wesleyan University, 2008.

Mckenna, Marian C., Franklin Roosevelt and the Great Constitution War: The Court-Packing Crisis of 1937, New York, Fordham University Press, 2002, pp. 539-544.

MigliUCCI, Dario, «Intolerable, peligrosa, imprescindible: intelectuales y políticos estadounidenses ante la problemática de la propaganda en el periodo de entreguerras (1919-1939)», Rubrica Contemporanea 5, 10, diciembre 2016, pp. 45-64.

MigliUCCI, Dario, «Opinión pública y propaganda: su definición, interpretación y significado en los Estados Unidos de la primera postguerra (1918-1922)», Historia y Política, 40, 2018a, pp. 213-238.

MigliuCCI, Dario, «Control gubernamental de la opinión pública: prácticas y polémicas en la arena política estadounidense», en Antonio Niño Rodríguez y Juan Ignacio Rospir Zabala, Democracia y control de la opinión pública en el periodo de entreguerras, 1918-1939, Madrid, Polifemo, 2018b, pp. 289334.

MigliUCCI, Dario, «An undervalued witch-hunt: Reassessing the nature and the impact of the 1930s struggle against un-American activities», American Communist History, 20, 1-2, 2021, pp. 73-94.

Mock, James R. y LARSON, Cedric, Words That Won the War. The Story of the Committee on Public Information, 1917-1919, New York, Russell \& Russell, 1939.

Monticone, Paul, «"Useful Cinema”, of What Use? Assessing the Role of Motion Pictures in the Largest Public Relations Campaign of the 1920s», Cinema Journal, 54, 4, verano de 2015, pp. 74-99.

Moser, John E., «"Gigantic Engines of Propaganda”: The 1941 Senate Investigation of Hollywood», Historian, 63, 4, 2001, pp. 731-752.

Moss, David, Bolton, Cole y Kintgen, Eugene, «The Pecora Hearings», Harvard Business School Case, 9, 711-046, December 2010.

NeuzIL, Mark, «Hearst, Roosevelt, and the Muckrake Speech of 1906: A New Perspective», Journalism and Mass Communication Quarterly, 73, 1, primavera de 1996, pp. 29-39.

Perino, Michael A., The Hellhound of Wall Street: How Ferdinand Pecora's Investigation of the Great Crash Forever Changed American Finance, New York, Penguin Press, 2010. 
La lucha institucional contra la propaganda de las grandes empresas estadounidenses ...

Peroncini, Gianfranco, La nascita dell'impero americano. 1934-1936: la Commissione Nye e l'intreccio industriale, militare e politico che ha governato il mondo, Milano, Mursia, 2014.

Perros, George P., Preliminary Inventory of the Records of the Select Committee on Lobbying activities, 1949-1950, The National Archives, National Archives and Records Service, General Services Administration, Washington, 1956.

SAYre, J. L.: «Gerald Nye: "Essentially Negative”», en Salter, J. T., Public Men: In and Out of Office, Chapel Hill, The University of North Carolina Press, 1946, pp. 127-146.

Sbardellati, John, «Brassbound G-Men and Celluloid Reds: the FBI's Search for Communist Propaganda in Wartime Hollywood», Film History, 20, 4, 2008, pp. 412-436.

SugÁR, András, «Brief History of Market Regulation Primarily from the Point of View of Price Regulation», Society and Economy, 33, 2, 2011, pp. 321-345.

ThIEME, Theodore F., The Germans in Politics; or How the Germans are Used by Big Business to Pull its Chestnuts out of the Fire, Fort Wayne, The citizens league of Indiana, ¿1918?.

Thompson, Carl D., Confessions of the Power Trust, New York, E.P. Dutton \& Co., Inc., 1932.

Van Der Veer Hamilton, Virginia, Hugo Black: The Alabama Years, Baton Rouge, Louisiana State University Press, 1982.

Vaughn, Stephen L., Holding Fast the Inner Lines. Democracy, Nationalism, and the Committee on Public Information, Chapel Hill, The University of North Carolina Press, 1980.

Wolf, Thomas Phillip, Pederson, William D. y Daynes, Byron W., Franklin D. Roosevelt and Congress: The New Deal and its Aftermath, Armonk, N.Y., M.E. Sharpe, 2001.

\section{Datos del Autor}

Dario Migliucci es beneficiario de un contrato posdoctoral «Margarita Salas» en la Universidad Complutense de Madrid; actualmente está desempeñando tareas docentes y de investigación en la Sorbonne Université. Licenciado en Historia por la Universidad de Granada (premio nacional fin de carrera), desde 2015 ha sido contratado FPU en la Universidad Complutense, consiguiendo en 2019 el título de doctor internacional en Historia Contemporánea (premio extraordinario de doctorado). También ha sido becario de investigación en la Universidad de Granada y en el CSIC, e investigador posdoctoral en la Universidad Nacional Autónoma de México. A lo largo de los últimos años ha estado investigando, por un lado, las representaciones desde Occidente del conflicto árabe-israelí (1947-1967); y, por otro, la respuesta al desafío de la propaganda de los políticos estadounidenses durante el periodo de entreguerras (1918-1941). Los resultados de sus inves- 
tigaciones han sido publicados en capítulos de libros y en distintas revistas académicas de impacto (JCR, SCOPUS, etc.), tanto en España como en el extranjero. Recientemente ha coordinado el libro (en coedición con Lucía López-Rodríguez) El conflicto humano: orígenes, dinámicas, secuelas y resolución de los conflictos contemporáneos (Sanz y Torres, 2021). Ha realizado estancias de investigación y periodos de movilidad académica en diversas instituciones de España, México, Reino Unido, Israel, Francia y Estados Unidos. 